\title{
Approximation of the Ornstein-Uhlenbeck local time by harmonic oscillators
}

\author{
JOSÉ R. LEÓN ${ }^{1}$ and GONZALO PERERA ${ }^{2}$ \\ ${ }^{1}$ Departamento de Matemáticas, Facultad de Ciencias, Universidad Central de Venezuela, \\ Caracas, Venezuela.E-mail: jleon@euler.ciens.ucv.ve \\ ${ }^{2}$ Centro de Matemática, Universidad de la República, Montevideo, Uruguay. \\ E-mail: gperera@fing.edu.uy
}

We consider a particle of mass $1 / \beta$ submitted to the action of an harmonic oscillator. If we add a white-noise external force, it is well known that the trajectories of the particle, for $\beta$ tending to infinity, converge to an Ornstein-Uhlenbeck process. Using the number of crossings of the particle with a fixed level $u$, we construct a consistent estimator of the Ornstein-Uhlenbeck local time, giving an estimate of the speed of this convergence.

Keywords: crossings; local time; diagram formula; mixing processes

\section{Introduction}

Wang and Uhlenbeck (1945) study the equation of the harmonic oscillator driven by white noise $\mathrm{d} W_{t}$ :

$$
x^{\prime \prime}(t)+\beta x^{\prime}(t)+\theta^{2} x(t)=\gamma \mathrm{d} W_{t} .
$$

If the initial conditions are suitably chosen, this equation has a stationary Gaussian solution with spectral density given by

$$
f(\lambda)=\frac{\gamma^{2}}{2 \pi\left[\left(\lambda^{2}-\theta^{2}\right)^{2}+\beta^{2} \lambda^{2}\right]} .
$$

From this formula it is trivial to show that the variance of the process, $\lambda_{0}$, and its second spectral moment, $\lambda_{2}$, are

$$
\lambda_{0}=\frac{\gamma^{2}}{2 \beta \theta^{2}} \quad \text { and } \quad \lambda_{2}=\frac{\gamma^{2}}{2 \beta} .
$$

Denoting by $N_{t}^{x}(u)$ the number of crossings of process $x$ with level $u$ up to time $t$, i.e.

$$
N_{t}^{x}(u)=\#\left\{0 \leqslant s \leqslant t: x_{s}=u\right\},
$$

we obtain, using Rice's formula of order 1 (see Rice 1945) that:

$$
\mathrm{E} N_{t}^{x}(u)=\frac{t}{\pi} \theta \exp \left(-\frac{u^{2}}{2 \lambda_{0}}\right)
$$


Thus the expectation of the number of zeros does not depend on $\beta$ and this random variable provides an unbiased estimator for frequency $\theta$.

Another physical model equivalent to the harmonic oscillator is the RLC (where R denotes a resistance, $\mathrm{L}$ an inductance and $\mathrm{C}$ a condenser) circuit considered by Rice (see Rice 1945). The equation in this case is

$$
L I^{\prime \prime}(t)+R I^{\prime}(t)+\frac{1}{C} I(t)=\gamma \mathrm{d} W_{t},
$$

where the 'frequency' is $\theta^{2}=1 / C L$ and $\beta=R / L$. In his seminal paper, in which he proved formula (1.2), Rice (1945, p. 57) wrote:

There are experiments which indicate the possibility of the formulas [(1.2)] breaking down in some cases. Prof. Uhlenbeck has pointed out that if a very broad band fluctuation current be forced to flow through a circuit consisting of a condenser, $\mathrm{C}$, in parallel with a series combination of inductance $\mathrm{L}$, and resistance, R, equation [(1.2)] says that the expected number of zeros per second of the current, I, flowing through R (and L) is independent of R. ... Curves are available for this motion and it seems that their character depends greatly upon the pressure. Unfortunately, it is difficult to tell from the curves whether the expected number of zeros is independent of the pressure.

Nelson (1968, p. 65) found that if the parameters of the equation satisfy certain restrictions, then the asymptotic behaviour (for $\beta$ going to infinity) of the solution can be determined. Using the law of the equipartition of energy, he obtained

$$
\gamma^{2}=\beta^{2} D
$$

(here we take $D=1$ ). On the other hand, he imposed

$$
\lim _{\beta \rightarrow \infty} \frac{\theta^{2}}{\beta}=\text { const. }
$$

(we will take $\theta^{2}:=\beta \omega^{2}$ ). Under these conditions equation (1.1) becomes

$$
x^{\prime \prime}(t)+\beta x^{\prime}(t)+\beta \omega^{2}(t)=\beta \mathrm{d} W_{t} .
$$

We will denote by $x(t, \beta)$ its stationary solution. Denoting by $f(\lambda, \beta)$ its spectral density, we have

$$
f(\lambda, \beta)=\frac{\beta^{2}}{2 \pi\left|-\lambda^{2}+\mathrm{i} \beta \lambda+\beta \omega^{2}\right|^{2}} .
$$

Therefore,

$$
\lim _{\beta \rightarrow \infty} f(\lambda, \beta)=\frac{1}{2 \pi\left(\lambda^{2}+\omega^{4}\right)}=g(\lambda) .
$$

The function $g(\lambda)$ is the spectral density of the stationary solution of the Ornstein-Uhlenbeck equation

$$
\mathrm{d} P_{s}+\omega^{2} P_{s}=\mathrm{d} W_{s}
$$

According to this, Nelson (1968) gave a rigorous proof that

$$
\lim _{\beta \rightarrow \infty} x(t, \beta)=P_{t} \text { a.s. }
$$


uniformly over compact intervals (see Nelson's Theorem 10.1). Therefore, the solution of equation (1.3) provides an estimate of the Ornstein-Uhlenbeck process which has been called the Smoluchowski approximation (see Karatzas and Shreve 1991, p. 362). For other classical estimates of the Ornstein-Uhlenbeck process and an excellent account of its origin, we refer the reader to Jacobsen (1996).

Using Rice's formula for the process $x(t, \beta)$, we obtain

$$
\mathrm{E}\left\{N_{t}^{x(\cdot, \beta)}(u)\right\}=\frac{t}{\pi} \sqrt{\beta} \omega \exp \left(-u^{2} \omega^{2}\right) .
$$

The process $P_{t}$ crosses all levels infinitely many times. Moreover, it has a bicontinuous local time $L_{t}^{P}(u)$, for almost every path (see Berman 1969; Revuz and Yor 1991, Chapter VI), defined as

$$
L_{t}^{P}(u)=\lim _{\varepsilon \rightarrow 0} \frac{\left|\left\{s \leqslant t:\left|P_{s}-u\right|<2 \varepsilon\right\}\right|}{2 \varepsilon} .
$$

A simple computation gives

$$
\mathrm{E}\left\{L_{t}^{P}(u)\right\}=t \frac{\omega}{\sqrt{\pi}} \exp \left(-u^{2} \omega^{2}\right)
$$

Hence we obtain

$$
\left(\frac{\pi}{\beta}\right)^{1 / 2} \mathrm{E}\left\{N_{t}^{x(\cdot, \beta)}(u)\right\}=\mathrm{E}\left\{L_{t}^{P}(u)\right\} .
$$

It is natural to conjecture that

$$
\lim _{\beta \rightarrow \infty}\left(\frac{\pi}{\beta}\right)^{1 / 2} N_{t}^{x(\cdot ; \beta)}(u)=L_{t}^{P}(u) \quad \text { in } L^{2}(\Omega) .
$$

In Theorem 1 we will prove this result. In Theorem 2 we give an estimate of the speed of this convergence. More precisely, let $f$ be a smooth real function: we will show in Theorem 2 that there is a Brownian motion $B$ independent of $W$ and a positive constant $\sigma$ such that

$$
\sqrt{\beta} \int_{-\infty}^{\infty} f(u)\left(\left(\frac{\pi}{\beta}\right)^{1 / 2} N_{t}^{x(\cdot, \beta)}(u)-L_{t}^{P}(u)\right) \mathrm{d} u \rightarrow \sigma \int_{0}^{t} f\left(P_{s}\right) \mathrm{d} B_{S}
$$

weakly when $\beta$ goes to infinity.

There are some related results in the literature. Indeed, several papers study the secondorder approximation to the Brownian local times. Borodin (1989) provides more exhaustive information concerning this kind of problem.

The regularization of an irregular process $P$ by convolution with an approximated identity has been succesfully used for the estimation of the local time of $P$. If $P$ is the Brownian motion (see Wschebor 1985), a stationary Gaussian process (see Azaïs and Florens 1987) or a diffusion process (see Azaiis (1989), then the number of crossings of the regularized processes (suitably normalized) converges to the local time of $P$ as in our Theorem 1. In that context we can also find some results analogous to our Theorem 2 (see Berzin et al. 1998). However, there are some substantial technical differences between that 
context and ours, mostly related to the fact that in our case the approximating process $x(\cdot, \beta)$ corresponds to the convolution of $P$ with a non-compactly supported kernel.

\section{Notation and $L^{2}(\Omega)$-convergence}

Let us define $P_{t}^{\beta}=x(t, \beta)$ and $V_{t}^{\beta}=x^{\prime}(t, \beta)$. The equation for the harmonic oscillator (1.1) is equivalent to the following system:

$$
\begin{aligned}
& \mathrm{d} P_{t}^{\beta}=V_{t}^{\beta} \mathrm{d} t, \quad P_{0}^{\beta}=p_{0}, \\
& \mathrm{~d} V_{t}^{\beta}=-\beta V_{t}^{\beta} \mathrm{d} t-\beta \omega^{2} P_{t}^{\beta} \mathrm{d} t+\beta \mathrm{d} W_{t}, \quad V_{0}^{\beta}=v_{0} .
\end{aligned}
$$

For the sake of simplicity, we will choose as initial conditions $p_{0}, v_{0}$ independent, centred and Gaussian random variables with variances to be made precise later on. Let us first fix some notation:

$$
X_{t}^{\beta}=\sqrt{2} \omega P_{t}^{\beta}, \quad Y_{t}^{\beta}=\sqrt{\frac{2}{\beta}} V_{t}^{\beta}=\frac{\dot{X}_{t}^{\beta}}{\sqrt{\beta \omega^{2}}} .
$$

We will choose the initial conditions $\left(X_{0}, Y_{0}\right)$ to be a standard Gaussian vector, independent of $W$. With that choice it is easy to see that $\left(X_{t}^{\beta}, Y_{t}^{\beta}\right)$ is a stationary Gaussian process.

Remark. It is easy to see that $N_{t}^{X^{\beta}}(u)=N_{t}^{P^{\beta}}(u / \sqrt{2} \omega), L_{t}^{X}(u)=(1 / \sqrt{2} \omega) L_{t}^{P}(u / \sqrt{2} \omega)$. Therefore, there is a difference of a factor $\sqrt{2} \omega$ in the normalization of the results to be presented in this section with respect to their statement in Section 1.

Given a standard Brownian motion $M$, we will denote by $U^{M}(a)$ the OrnsteinUhlenbeck process of parameter $a$ generated by $M$, i.e.

$$
U_{t}^{M}(a)=\exp (-a t) \int_{0}^{t} \exp (a s) \mathrm{d} M_{s} .
$$

Define

$$
X_{t}=\exp \left(-\omega^{2} t\right) X_{0}+\sqrt{2 \omega^{2}} U_{t}^{W}\left(\omega^{2}\right) .
$$

With this new notation, Nelson's theorem states that:

$$
X_{t}^{\beta}(t) \rightarrow X_{t} \text { a.s. }
$$

uniformly over compact intervals.

Using well-known results for Gaussian processes (see Wschebor 1985, p 14) it follows that, for each level $u, P\left\{\exists s \in[0, t]: X_{s}^{\beta}=u, \dot{X}_{s}^{\beta}=0\right\}=0$, and therefore $N_{t}^{X^{\beta}}(u)$ is a.s. finite.

On the other hand, we will denote by $L_{t}^{X}(u)$ the local time of $X$ at level $u$ (up to time $t$ ); it can be taken as an a.s. continuous function of $(t, u)$. 
Useful representations of both $N_{t}^{X^{\beta}}(u)$ and $L_{t}^{X}(u)$ are given by the Banach-Kac formulae (see Nualart and Wschebor 1991, Lemma 1.1):

$$
\begin{aligned}
N_{t}^{X^{\beta}}(u) & =\lim _{\delta \rightarrow 0^{+}} \frac{1}{2 \delta} \int_{0}^{t} 1_{(u-\delta, u+\delta)}\left(X_{s}^{\beta}\right)\left|\dot{X}_{s}^{\beta}\right| \mathrm{d} s, \\
L_{t}^{X}(u) & =\lim _{\delta \rightarrow 0^{+}} \frac{1}{2 \delta} \int_{0}^{t} 1_{(u-\delta, u+\delta)}\left(X_{s}\right) \mathrm{d} s .
\end{aligned}
$$

A final remark on notation: we will say that a sequence of random processes converges f.d. if their finite-dimensional distributions converge.

Theorem 1. For each level $u$, we have

$$
\lim _{\beta \rightarrow \infty} \sqrt{\frac{\pi}{2 \beta w^{2}}} N_{t}^{X^{\beta}}(u)=L_{t}^{X}(u) \quad\left(\text { in } L^{2}(\Omega)\right) .
$$

Proof. Applying Rice's formula (see Wschebor 1985, p. 43), we obtain

$$
\mathrm{E}\left\{\sqrt{\frac{\pi}{2 \beta w^{2}}} N_{t}^{X^{\beta}}(u)\right\}=\mathrm{E}\left\{L_{t}^{X}(u)\right\} .
$$

Therefore, it is enough to see that

$$
\begin{gathered}
\lim _{\beta \rightarrow \infty} \frac{\pi}{2 \beta w^{2}} \mathrm{E}\left\{N_{t}^{X^{\beta}}(u)\left[N_{t}^{X^{\beta}}(u)-1\right]\right\}=\mathrm{E}\left\{\left[L_{t}^{X}(u)\right]^{2}\right\}, \\
\lim _{\beta \rightarrow \infty} \sqrt{\frac{\pi}{2 \beta w^{2}}} \mathrm{E}\left\{N_{t}^{X^{\beta}}(u) L_{t}^{X}(u)\right\}=\mathrm{E}\left\{\left[L_{t}^{X}(u)\right]^{2}\right\} .
\end{gathered}
$$

The proof of (2.1) is obtained as follows. Using Rice's second-order formula (see Wschebor 1985 , p. 61), we obtain

$$
\frac{\pi}{2 \beta w^{2}} \mathrm{E}\left\{N_{t}^{X^{\beta}}(u)\left[N_{t}^{X^{\beta}}(u)-1\right]\right\}=\int_{0}^{t} \Psi^{\beta}(s) \mathrm{d} s,
$$

for

$$
\Psi^{\beta}(s)=\frac{(t-s) \exp \left(-\frac{u^{2}}{1+\rho^{\beta}(s)}\right)}{4 \beta w^{2} \sqrt{1-\left(\rho^{\beta}(s)\right)^{2}}} \mathrm{E}\left\{\left|\xi_{s}^{\beta}-\frac{u \dot{\rho}^{\beta}(s)}{1+\rho^{\beta}(s)}\right|\left|\xi_{s}^{\beta}+\frac{u \dot{\rho}^{\beta}(s)}{1+\rho^{\beta}(s)}\right|\right\},
$$

where $\left(\xi_{s}^{\beta}, \xi_{s}^{\beta}\right)$ is a centred Gaussian vector with

$$
\operatorname{var}\left(\xi_{s}^{\beta}\right)=\operatorname{var}\left(\xi_{s}^{\beta}\right)=-\ddot{\rho}^{\beta}(0)-\frac{\dot{\rho}^{\beta}(s)}{\left[1-\left(\rho^{\beta}(s)\right)^{2}\right]}
$$

and 


$$
\operatorname{cov}\left(\xi_{s}^{\beta}, \xi_{s}^{\beta}\right)=-\ddot{\rho}^{\beta}(s)-\frac{\rho^{\beta}(s) \dot{\rho}^{\beta}(s)}{\left[1-\left(\rho^{\beta}(s)\right)^{2}\right]},
$$

and $\rho^{\beta}$ stands for the correlation of $X^{\beta}$.

It is easy to check that

$$
\begin{aligned}
& \rho^{\beta}(s)=\left\{\frac{\mathrm{e}^{\lambda_{1}^{\beta} s}}{\lambda_{1}^{\beta}}-\frac{\mathrm{e}^{\lambda_{2}^{\beta} s}}{\lambda_{2}^{\beta}}\right\} \frac{\left(\lambda_{2}^{\beta}-\lambda_{1}^{\beta}\right)}{\beta-4 w^{2}} w^{2}, \\
& \dot{\rho}^{\beta}(s)=\left\{\mathrm{e}^{\lambda_{1}^{\beta^{\beta}}}-\mathrm{e}^{\lambda_{2}^{\beta} s}\right\} \frac{\left(\lambda_{2}^{\beta}-\lambda_{1}^{\beta}\right)}{\beta-4 w} w^{2}, \\
& \ddot{\rho}^{\beta}(s)=\left\{\lambda_{1}^{\beta} \mathrm{e}^{\lambda_{1}^{\beta} s}-\lambda_{2}^{\beta} \mathrm{e}^{\lambda_{2}^{\beta} s}\right\} \frac{\left(\lambda_{2}^{\beta}-\lambda_{1}^{\beta}\right)}{\beta-4 w^{2}} w^{2},
\end{aligned}
$$

where $\lambda_{i}^{\beta}, i=1,2$, are the eigenvalues of $(1.1)$, i.e.

$$
\begin{aligned}
& \lambda_{1}^{\beta}=\frac{-\beta-\sqrt{\beta\left(\beta-4 \omega^{2}\right)}}{2} \\
& \lambda_{2}^{\beta}=\frac{-\beta+\sqrt{\beta\left(\beta-4 \omega^{2}\right)}}{2} .
\end{aligned}
$$

Denoting by $\rho(s)=\mathrm{e}^{-w^{2} s}$ the correlation function of $X$, it follows that, for all $s \geqslant 0$,

$$
\lim _{\beta \rightarrow \infty} \rho^{\beta}(s)=\rho(s), \quad \lim _{\beta \rightarrow \infty} \dot{\rho}^{\beta}(s)=\dot{\rho}(s),
$$

and that the Gaussian vector

$$
\frac{1}{\omega \sqrt{\beta}}\left(\xi_{s}^{\beta}-\frac{u \dot{\rho}^{\beta}(s)}{1+\rho^{\beta}(s)}, \xi_{s}^{\beta}-\frac{u \dot{\rho}^{\beta}(s)}{1+\rho^{\beta}(s)}\right)
$$

converges in law to a standard Gaussian vector in $\mathbb{R}^{2},(\xi, \zeta)$. This implies in turn that

$$
\lim _{\beta \rightarrow \infty} \frac{1}{4 \beta \omega^{2}} \mathrm{E}\left\{\left|\xi_{s}^{\beta}-\frac{u \dot{\rho}^{\beta}(s)}{1+\rho^{\beta}(s)}\right|\left|\xi_{s}^{\beta}+\frac{u \dot{\rho}^{\beta}(s)}{1+\rho^{\beta}(s)}\right|\right\}=\frac{1}{4} \mathrm{E}(|\xi \| \xi|)=\frac{\mathrm{E}(|\xi|)^{2}}{4}=\frac{1}{2 \pi}
$$

and, defining

$$
\Psi(s)=\frac{(t-s) \exp \left(\frac{-u^{2}}{1+\rho(s)}\right)}{2 \pi \sqrt{1-\rho(s)^{2}}},
$$

it follows that, for each $s>0, \Psi^{\beta}(s)$ converges to $\Psi(s)$ as $\beta$ goes to infinity. On the other hand, it is very easy to see that $\mathrm{E}\left\{\left[L_{t}^{X}(u)\right]^{2}\right\}=\int_{0}^{t} \Psi(s) \mathrm{d} s$. As is well known, if

$$
0 \leqslant \Psi^{\beta}(s) \leqslant \eta^{\beta}(s), \quad \forall s>0 ; \quad \lim _{\beta \rightarrow \infty} \eta^{\beta}(s)=\eta(s), \quad \forall s>0 ; \quad \eta^{\beta}, \eta \in L^{1}((0, t]) ;
$$




$$
\lim _{\beta \rightarrow \infty} \int_{0}^{t} \eta^{\beta}(s) \mathrm{d} s=\int_{0}^{t} \eta(s) \mathrm{d} s ; \quad \lim _{\beta \rightarrow \infty} \Psi^{\beta}(s)=\Psi(s) \quad \forall s>0,
$$

then

$$
\lim _{\beta \rightarrow \infty} \int_{0}^{t} \Psi^{\beta}(s) \mathrm{d} s=\int_{0}^{t} \Psi(s) \mathrm{d} s
$$

Therefore, to prove (2.1), it sufficies to find $\eta^{\beta}, \eta$ as above.

But, using Cauchy-Schwarz and (2.3), we have

$$
0 \leqslant \Psi^{\beta}(s) \leqslant \frac{(t-s) \exp \left(\frac{-u^{2}}{1+\rho^{\beta}(s)}\right)}{4 \beta w^{2} \sqrt{1-\left(\rho^{\beta}(s)\right)^{2}}}\left[\operatorname{var}\left(\xi_{s}^{\beta}\right)+\frac{u^{2}\left(\dot{\rho}^{\beta}(s)\right)^{2}}{\left(1+\rho^{\beta}(s)\right)^{2}}\right]
$$

Elementary computation shows that $\Psi^{\beta}(s) \leqslant \tau^{\beta}(s)+v^{\beta}(s)$, with

$$
\tau^{\beta}(s)=K_{2}\left\{\frac{1+\rho^{\beta}(s)}{\sqrt{1-\rho^{\beta}(s)}}-\frac{(\dot{\rho} \beta(s))^{2}}{\left[1-\rho^{\beta}(s)\right]^{3 / 2} \beta w^{2}}\right\}, \quad v^{\beta}(s)=\frac{K_{3}\left(\dot{\rho}^{\beta}(s)\right)^{2}}{\beta \sqrt{1-\rho^{\beta}(s)}} .
$$

Take $\eta^{\beta}=\tau^{\beta}+v^{\beta}$ and define $\tau(s)=(1+\rho(s)) / \sqrt{1-p(s)}$, since $\rho(s)=\mathrm{e}^{-w^{2} s}$; some elementary calculus (where we use the equality $\ddot{\rho}^{\beta}=-\beta\left(w^{2} \rho^{\beta}+\dot{\rho}^{\beta}\right)$ ) shows that

$$
\lim _{\beta-\infty} \tau^{\beta}=\tau \text { a.e., } \quad \tau^{\beta}, \tau \text { integrable; } \quad \lim _{\beta \rightarrow \infty} \int_{0}^{t} \tau^{\beta}(s) \mathrm{d} s=\int_{0}^{t} \tau(s) \mathrm{d} s
$$

and

$$
\lim _{\beta \rightarrow \infty} v^{\beta}=0 \text { a.e., } v^{\beta} \text { integrable; } \quad \lim _{\beta \rightarrow \infty} \int_{0}^{t} v^{\beta}(s) \mathrm{d} s=0,
$$

and (2.1) follows.

In order to prove (2.2), it is easy to see, from the Banach-Kac formulae, that

$$
\sqrt{\frac{\pi}{2 \beta \omega^{2}}} \mathrm{E}\left\{N_{t}^{X^{\beta}}(u) L_{t}^{X}(u)\right\}=\int_{0}^{t} \mu^{\beta}(s) \mathrm{d} s,
$$

where $\mu^{\beta}(s)=\sqrt{\left(\pi / 2 \beta \omega^{2}\right)} \mathrm{E}\left\{\left|\dot{X}_{s}^{\beta}\right| / X_{s}^{\beta}=X_{s}=u\right\} ; \lim _{\beta \rightarrow \infty} \mu^{\beta}(s)=\psi(s)$, for all $s>0$, and hence, by Fatou's lemma, $\mathrm{E}\left\{\left(L_{t}^{X}(u)\right)^{2}\right\} \leqslant \liminf _{\beta \rightarrow \infty} \sqrt{\left(\pi / 2 \beta \omega^{2}\right)} \mathrm{E}\left\{N_{t}^{X^{\beta}}(u) L_{t}^{X}(u)\right\}$. The opposite inequality is obtained from the Cauchy-Schwarz inequality and (2.1).

\section{Weak convergence results}

We will begin with two elementary remarks that play an essential role in the proof of Theorem 2.

First, let $M$ be a standard Brownian motion and $U^{M}(a)$ the Ornstein-Uhlenbeck process of parameter $a$ generated by $M$. Consider $Z^{M}(a)$, a stationary Ornstein-Uhlenbeck process of parameter $a$ generated by $M$, defined by 


$$
Z_{t}^{M}(a)=\exp (-a t) Z_{0}+\sqrt{2 a} U_{t}^{M}(a)
$$

where $Z_{0}$ is any $N(0,1)$ random variable independent of $M$. A straightforward computation shows that

$$
M_{t}(a)=\frac{Z_{t}^{M}(a)-Z_{0}}{\sqrt{2 a}}+\sqrt{\frac{a}{2}} \int_{0}^{t} Z_{s}^{M}(a) \mathrm{d} s .
$$

we will refer to this equation as the $O U$ inversion formula.

The second remark is a formula that enables us to integrate a function with respect to the number of crossings. Let $I$ be a bounded interval, $g$ a $C^{1}(I)$ function, $f$ continuous and bounded in $\mathbb{R}$, and denote by $N_{I}^{g}(u)$ the number of roots of the equation $g(t)=u, t \in I$. Then

$$
\int_{-\infty}^{\infty} f(u) N_{I}^{g}(u) \mathrm{d} u=\int_{I} f(g(t))\left|g^{\prime}(t)\right| \mathrm{d} t
$$

(see Nualart and Wschebor (1991, p. 88).

Define

$$
V(\beta)=\sqrt{\beta} \int_{-\infty}^{\infty} f(u)\left(\sqrt{\frac{\pi}{2 \beta \omega^{2}}} N_{t}^{X(\beta)}(u)-L_{t}^{X}(u)\right) \mathrm{d} u .
$$

By using the previous formula, we get the decomposition

$$
V(\beta)=\sqrt{\beta} \int_{0}^{t} f\left(X_{s}^{\beta}\right) g\left(Y_{s}^{\beta}\right) \mathrm{d} s+\sqrt{\beta} \int_{0}^{t}\left[f\left(X_{s}^{\beta}\right)-f\left(X_{s}\right)\right] \mathrm{d} s:=J(\beta)+R(\beta),
$$

where

$$
J(\beta)=\sqrt{\beta} \int_{0}^{t} f\left(X_{s}^{\beta}\right) g\left(Y_{s}^{\beta}\right) \mathrm{d} s, \quad R(\beta)=\sqrt{\beta} \int_{0}^{t}\left[f\left(X_{s}^{\beta}\right)-f\left(X_{s}\right)\right] \mathrm{d} s
$$

and

$$
g(x)=\sqrt{\frac{\pi}{2}}|x|-1 .
$$

We will show below that $R(\beta) \rightarrow 0$ in $L^{2}(\Omega)$ when $\beta$ goes to infinity. Hence, to obtain the asymptotic distribution of $V(\beta)$, it is enough to consider only $J(\beta)$.

We must first study the limit of $\operatorname{var}(J(\beta))$, but since $\mathrm{E}(J(\beta))=0$, we obtain

$$
\lim _{\beta \rightarrow \infty} \operatorname{var}(J(\beta))=\lim _{\beta \rightarrow \infty} \mathrm{E}\left[\sqrt{\beta} \int_{0}^{t} f\left(X_{s}^{\beta}\right) g\left(Y_{s}^{\beta}\right) \mathrm{d} s\right]^{2} ;
$$

we will study this limit for a more general function $g$.

We will use some basic properties of Gaussian chaos: define the Hermite polynomials $\left(H_{n}: n \in \mathbb{N}\right)$ by 


$$
\exp \left(t x-\frac{t^{2}}{2}\right)=\sum_{n=0}^{\infty} H_{n}(x) \frac{t^{n}}{n !}
$$

These polynomials form an orthogonal system for the standard Gaussian measure $\mu$. Therefore, if $h \in L^{2}(\mu)$ we will expand $h(x)=\sum_{n=0}^{\infty} \hat{h}_{n} H_{n}(x)$ and we have $\|h\|_{L^{2}(\mu)}^{2}=$ $\sum_{n=0}^{\infty} \hat{h}_{n}^{2} n !$.

Mehler's formula states that if $(X, Y)$ is a Gaussian random vector having correlation $\rho$, and if $h, k \in L^{2}(\mu)$, then

$$
\mathrm{E}[h(X) k(Y)]=\sum_{n=0}^{\infty} \hat{h}_{n} \hat{k}_{n} n ! \rho^{n}
$$

We will first state an auxiliary result.

Lemma 1. For any $s>0$, we have $\left(X_{0}, Y_{0}, X_{s / \beta}^{\beta}, Y_{s / \beta}^{\beta}\right) \stackrel{\mathscr{C}}{\rightarrow}\left(X_{0}, Y_{0}, X_{0}, Y_{s}\right)$, and the limit is a Gaussian vector with $\mathrm{E}\left(X_{0} Y_{s}\right)=0, \mathrm{E}\left(Y_{0} Y_{s}\right)=\mathrm{e}^{-s}$.

Proof. Observe that $\left(X_{0}, Y_{0}, X_{s / \beta}^{\beta}, Y_{s / \beta}^{\beta}\right)$ is a Gaussian vector, and compute covariances using the fact that, for $0 \leqslant u \leqslant s$,

$\mathrm{E}\left(X_{u}^{\beta} X_{s}^{\beta}\right)=\rho^{\beta}(s-u), \mathrm{E}\left(X_{u}^{\beta} Y_{s}^{\beta}\right)=\dot{\rho}^{\beta}(s-u), \mathrm{E}\left(Y_{u}^{\beta} X_{s}^{\beta}\right)=-\dot{\rho}^{\beta}(s-u), \mathrm{E}\left(Y_{u}^{\beta} Y_{s}^{\beta}\right)=-\ddot{\rho}^{\beta}(s-u)$.

We shall now compute the asymptotic variance.

Proposition 1. Let $h: \mathbb{R} \rightarrow \mathbb{R}$ be a continuous even function such that $\mathrm{E}\left[h(\xi)^{2}\right]<+\infty$, where $\xi$ is a standard Gaussian variable. Then

$$
\lim _{\beta \rightarrow \infty} \mathrm{E}\left[\sqrt{\beta} \int_{0}^{t} f\left(X_{s}^{\beta}\right) h\left(Y_{s}^{\beta}\right) \mathrm{d} s\right]^{2}=\sigma^{2} t \mathrm{E}\left[f^{2}\left(X_{0}\right)\right],
$$

where $\sigma^{2}=2 \int_{0}^{\infty} \mathrm{E}\left\{h\left(Y_{0}\right) h\left(Y_{s}\right)\right\} \mathrm{d} s$ and $Y$ is a stationary Ornstein-Uhlenbeck process of parameter 1 .

Proof. Using the fact that $\left(X_{s}^{\beta}, Y_{s}^{\beta}\right)$ is stationary and that if $\varphi$ is an even real function, then $\int_{0}^{t} \int_{0}^{t} \varphi(u-s) \mathrm{d} u \mathrm{~d} s=2 \int_{0}^{t}(t-s) \varphi(s) \mathrm{d} s$, we obtain

$$
\mathrm{E}\left[\sqrt{\beta} \int_{0}^{t} f\left(X_{s}^{\beta}\right) h\left(Y_{s}^{\beta}\right) \mathrm{d} s\right]^{2}=2 \beta \int_{0}^{t}(t-s) \mathrm{E}\left\{f\left(X_{0}\right) f\left(X_{s}^{\beta}\right) h\left(Y_{0}\right) h\left(Y_{s}^{\beta}\right)\right\} \mathrm{d} s:=K(\beta) .
$$

We can split our domain of integration into $[0, M / \beta]$ and $[M / \beta, t]$, obtaining 


$$
\begin{aligned}
K(\beta)= & 2 \beta \int_{0}^{M / \beta}(t-s) \mathrm{E}\left\{f\left(X_{0}\right) f\left(X_{s}^{\beta}\right) h\left(Y_{0}\right) h\left(Y_{s}^{\beta}\right)\right\} \mathrm{d} s \\
& +2 \beta \int_{M / \beta}^{t}(t-s) \mathrm{E}\left\{f\left(X_{0}\right) f\left(X \mathrm{f}_{s}^{\beta}\right) h\left(Y_{0}\right) h\left(Y_{s}^{\beta}\right)\right\} \mathrm{d} s \\
& :=K_{1}(\beta)+K_{2}(\beta) .
\end{aligned}
$$

Taken $u / \beta=s$ in $K_{1}(\beta)$, we obtain

$$
K_{1}(\beta)=2 \int_{0}^{M}\left(t-\frac{s}{\beta}\right) \mathrm{E}\left\{f\left(X_{0}\right) h\left(Y_{0}\right) f\left(X_{s / \beta}^{\beta}\right) h\left(Y_{s / \beta}^{\beta}\right)\right\} \mathrm{d} s .
$$

But

$$
\begin{aligned}
\left|\mathrm{E}\left\{f\left(X_{0}\right) h\left(Y_{0}\right) f\left(X_{s}^{\beta}\right) h\left(Y_{s}^{\beta}\right)\right\}\right| & \leqslant\left(\mathrm{E}\left\{f^{2}\left(X_{0}\right) h^{2}\left(Y_{0}\right)\right\}\right)^{1 / 2}\left(\mathrm{E}\left\{f^{2}\left(X_{s}^{\beta}\right) h^{2}\left(Y_{s}^{\beta}\right)\right\}\right)^{1 / 2} \\
& =\mathrm{E}\left(f^{2}\left(X_{0}\right)\right) \mathrm{E}\left(h^{2}\left(Y_{0}\right)\right) \leqslant C .
\end{aligned}
$$

Since the integrand is bounded, we can apply Lemma 1 and the dominated convergence theorem to obtain

$$
\lim _{\beta \rightarrow \infty} K_{1}(\beta)=2 t \mathrm{E}\left\{f^{2}\left(X_{0}\right)\right\} \int_{0}^{M} \mathrm{E}\left\{h\left(Y_{0}\right) h\left(Y_{s}\right)\right\} \mathrm{d} s .
$$

Given $\varepsilon>0$, we will choose $M$ in such a way that

$$
C^{*} \mathrm{e}^{-M}<\varepsilon
$$

where $C^{*}=\mathrm{E}\left\{f^{2}\left(X_{0}\right)\right\} \sum_{l=1}^{\infty} \hat{h}(2 l)^{2}(2 l)$ ! (observe that since $h$ is even, $\hat{h}(2 l+1)=0 \forall l$ ). Thus, by Mehler's formula, we have

$$
\begin{aligned}
\mid \mathrm{E}\left\{f^{2}\left(X_{0}\right)\right\} \int_{M}^{\infty} \mathrm{E}\left\{h\left(Y_{0}\right) h\left(Y_{s}\right) \mathrm{d} s \mid\right. & \leqslant \mathrm{E}\left\{f^{2}\left(X_{0}\right)\right\} \int_{M}^{\infty} \sum_{l=1}^{\infty} \hat{h}_{2 l}^{2}(2 l) !\left(\mathrm{E}\left\{Y_{0} Y_{s}\right\}\right)^{2 l} \mathrm{~d} s \\
& \leqslant \mathrm{E}\left\{f^{2}\left(X_{0}\right)\right\} \sum_{l=1}^{\infty} \hat{h}_{2 l}^{2}(2 l) ! \mathrm{e}^{-M}=C^{*} \mathrm{e}^{-M}<\varepsilon .
\end{aligned}
$$

For $K_{2}(\beta)$, we apply Lemma 2 (see the Appendix) and obtain

$$
\lim _{M \rightarrow \infty} \limsup _{\beta \rightarrow \infty}\left|K_{2}(\beta)\right|=0
$$

From (3.2), (3.3) and (3.4) we obtain

$$
\limsup _{\beta \rightarrow \infty} \mid \beta \int_{0}^{t} \mathrm{E}\left\{f\left(X_{0}\right) h\left(Y_{0}\right) f\left(X_{s}^{\beta}\right) h\left(Y_{s}^{\beta}\right\} \mathrm{d} s-2 t \mathrm{E}\left\{f\left(X_{0}\right)^{2}\right\} \int_{0}^{\infty} \mathrm{E}\left\{h\left(Y_{0}\right) h\left(Y_{s}\right)\right\} \mathrm{d} s \mid \leqslant \varepsilon .\right.
$$

Since $\varepsilon>0$ is arbitrary, the proposition follows.

We turn now to the main result of this section. 
Theorem 2. If $f$ is a real function with bounded and continuous second derivative, then

$$
\lim _{\beta \rightarrow \infty} \sqrt{\beta} \int_{-\infty}^{\infty} f(u)\left\{\sqrt{\frac{\pi}{2 \beta \omega^{2}}} N_{t}^{X^{\beta}}(u)-L_{t}^{X}(u)\right\} \mathrm{d} u=\sigma \int_{0}^{t} f\left(X_{s}\right) \mathrm{d} B_{s},
$$

where $B$ is a standard Brownian motion independent of $W, \sigma^{2}=2 \int_{0}^{\infty} \mathrm{E}\left\{g\left(Y_{0}\right) g\left(Y_{u}\right)\right\} \mathrm{d} u$ with $g(x)=\sqrt{(\pi / 2)}|x|-1$, and Y a stationary Ornstein-Uhlenbeck process of parameter 1 .

Proof. We will divide the proof into several steps:

Step 1. $\lim _{\beta \rightarrow \infty} R(\beta)=0$ (in $L^{2}$ ), where $R(\beta)=\sqrt{\beta} \int_{0}^{t}\left[f\left(X_{s}^{\beta}\right)-f\left(X_{s}\right)\right] \mathrm{d} s$.

Step 2. $\lim _{\beta \rightarrow \infty} \mathrm{E}\left\{J(\beta)^{2}\right\}=\sigma^{2} t \mathrm{E}\left\{f^{2}\left(X_{0}\right)\right\}$, where $J(\beta)=\sqrt{\beta} \int_{0}^{t} f\left(X_{s}^{\beta}\right) g\left(Y_{s}^{\beta}\right) \mathrm{d} s$.

Step 3. There is a stationary Ornstein-Uhlenbeck process of parameter $1, Z^{\beta}$, such that:

$$
\lim _{\beta \rightarrow \infty} \sqrt{\beta} \int_{0}^{t}\left\{g\left(Y_{s}^{\beta}\right)-g\left(Z_{-\lambda_{1}^{\beta} s}^{\beta}\right) \mathrm{d} s\right\}=0 \quad \forall t \quad\left(\text { in } L^{2}(\Omega)\right) .
$$

Step 4. The random process $C(\beta)_{y, t}=\left(W_{y}, \sqrt{\beta} \int_{0}^{t} g\left(Z_{-\lambda_{1}^{\beta} s}^{\beta}\right) \mathrm{d} s\right)$ converges weakly to $\left(W_{y}, \sigma B_{t}\right)$ (as a continuous process in $y$, f.d. with respect to ${ }^{-\lambda^{\beta}} t$ ) when $\beta$ goes to infinity.

Step 5. If

$$
V(\beta)=\sqrt{\beta} \int_{0}^{t} f\left(X_{s}^{\beta}\right) g\left(Y_{s}^{\beta}\right) \mathrm{d} s
$$

and

$$
V_{n}(\beta)=\sqrt{\beta} \sum_{n=1}^{[n t]} f\left(X_{\frac{i-1}{n}}^{\beta}\right) \int_{\frac{i-1}{n}}^{\frac{i}{n}} g\left(Y_{s}^{\beta}\right) \mathrm{d} s
$$

then

$$
\limsup _{n \rightarrow \infty} \limsup _{\beta \rightarrow \infty}\left\|V(\beta)-V_{n}(\beta)\right\|_{2}^{2}=0 .
$$

Assume that all these steps have been proved. Then, by step 4 and the fact that $X^{\beta}$ converges a.s. uniformly over compact intervals to $X$, we deduce that, for fixed $n$, when $\beta \rightarrow \infty, V_{n}(\beta)$ converges in distribution to $V_{n}$, where

$$
V_{n}=\sigma \sum_{i=1}^{[n t]} f\left(X_{\frac{i-1}{n}}\right)\left(B_{\frac{i}{n}}-B_{\frac{i-1}{n}}\right) .
$$

A straightforward computation shows that, when $n$ goes to infinity, $V_{n}$ converges in distribution to $\sigma \int_{0}^{t} f\left(X_{s}\right) \mathrm{d} B_{s}$. Then, step 5 implies in turn that $V(\beta)$ converges in distribution, for $\beta$ tending to infinity, to $\sigma \int_{0}^{t} f\left(X_{s}\right) \mathrm{d} B_{s}$. Therefore, by decomposition (3.1) and step 1 , the theorem is proved.

We have seen that it suffices to show steps 1 to 5 . We will present now their proofs.

Proof of step 1. By Taylor's formula, we have 


$$
\sqrt{\beta}\left(f\left(X_{s}^{\beta}-f\left(X_{s}\right)\right)=f^{\prime}\left(X_{s}\right) \sqrt{\beta}\left(X_{s}^{\beta}-X_{s}\right)+\frac{f^{\prime \prime}\left(\xi_{s}(\beta)\right)}{2} \frac{\left.\left[\sqrt{\beta}\left(X_{s}^{\beta}-X_{s}\right)\right)\right]^{2}}{\sqrt{\beta}}\right.
$$

then

$$
\mathrm{E}\left\{R^{2}(\beta)\right\} \leqslant 2\left\{\mathrm{E}\left[\left(\int_{0}^{t} f^{\prime}\left(X_{s}\right) \sqrt{\beta}\left(X_{s}^{\beta}-X_{s}\right) \mathrm{d} s\right)^{2}\right]\right\}+2\left\{\frac{1}{\beta} \mathrm{E}\left[\int_{0}^{t} \frac{f^{\prime \prime}\left(\xi_{s}(\beta)\right)}{2} \sqrt{\beta}\left(X_{s}^{\beta}-X_{s}\right)^{2} \mathrm{~d} s\right]^{2}\right\} .
$$

Using Jensen's inequality and the fact that $f^{\prime \prime}$ is bounded, we obtain that the second term is $O(1 / \beta)$.

For the first, we have

$$
\begin{aligned}
\mathrm{E}\left\{\left[\int_{0}^{t} f^{\prime}\left(X_{s}\right) \sqrt{\beta}\left(X_{s}^{\beta}-X_{s}\right) \mathrm{d} s\right]^{2}\right\} & =\int_{0}^{t} \int_{0}^{t} \mathrm{E}\left\{f^{\prime}\left(X_{s}\right) f^{\prime}\left(X_{r}\right) \sqrt{\beta}\left(X_{s}^{\beta}-X_{s}\right) \sqrt{\beta}\left(X_{r}^{\beta}-X_{r}\right) \mathrm{d} s \mathrm{~d} r\right\} \\
& :=\int_{0}^{t} \int_{0}^{t} \mu^{\beta}(s, r) \mathrm{d} s \mathrm{~d} r .
\end{aligned}
$$

By Hölder $\left|\mu^{\beta}(s, r)\right| \leqslant\left(\mathrm{E}\left(f^{\prime}\left(X_{0}\right)^{4}\right) v_{s}^{\beta} v_{r}^{\beta} \mathrm{E}\left(\xi^{4}\right)\right)^{1 / 2}$, where $v_{s}^{\beta}=2 \beta\left(1-\mathrm{E}\left(X_{s}^{\beta} X_{s}\right)\right)$ and $\xi$ is standard Gaussian; a direct computation shows that $v_{s}^{\beta} \rightarrow \omega^{2}+\omega^{2} \mathrm{e}^{-2 \omega^{2} s}$ when $\beta \rightarrow \infty$, uniformly in $s$. Indeed, it is easy to show that

$$
\begin{aligned}
v_{s}^{\beta}= & \exp \left(-\omega^{2} s\right) \frac{-\lambda_{2}^{\beta} \exp \left(\lambda_{1}^{\beta} s\right)+\lambda_{1}^{\beta} \exp \left(\lambda_{2}^{\beta} s\right)}{\lambda_{1}^{\beta}-\lambda_{2}^{\beta}} \\
& +\frac{2 \omega^{2} \beta}{\lambda_{1}^{\beta}-\lambda_{2}^{\beta}}\left(\frac{1-\exp \left(\left(\lambda_{1}^{\beta}-\omega^{2}\right) s\right)}{\omega^{2}-\lambda_{1}^{\beta}}+\frac{1-\exp \left(\left(\lambda_{2}^{\beta}-\omega^{2}\right) s\right)}{\omega^{2}-\lambda_{2}^{\beta}}\right) .
\end{aligned}
$$

This can be obtained as follows: first, use

$$
X_{s}=\exp \left(-\omega^{2} s\right) X_{0}+\sqrt{2 \omega^{2}} U_{s}^{W}\left(\omega^{2}\right)
$$

second, using the equation of the harmonic oscillator and elementary computations, compute the deterministic functions $A_{s}^{\beta}, B_{s}^{\beta}, C_{s}^{\beta}$ such that

$$
X_{s}^{\beta}=A_{s}^{\beta} X_{0}+B_{s}^{\beta} Y_{0}+C_{s}^{\beta}\left(U_{s}^{W}\left(-\lambda_{1}^{\beta}\right)-U_{s}^{W}\left(-\lambda_{2}^{\beta}\right)\right)
$$

and obtain the result by using the independence of $X_{0}, Y_{0}, W$ and the fact that

$$
\mathrm{E}\left(U_{s}^{W}(a) U_{s}^{W}(b)\right)=\frac{1-\exp (-(a+b) s)}{a+b} .
$$

Hence

$$
\left|\mu^{\beta}(s, r)\right| \leqslant C
$$

On the other hand, the vector

$$
W^{\beta}(s, r):=\left(X_{s}, X_{r}, \sqrt{\beta}\left(X_{s}^{\beta}-X_{s}\right), \sqrt{\beta}\left(X_{r}^{\beta}-X_{r}\right)\right)
$$


tends weakly to the Gaussian vector $\left(X_{s}, X_{r}, D_{s}, D_{r}\right)$. It follows that, when $\beta \rightarrow \infty$,

$$
\mu^{\beta}(s, r) \rightarrow \mu(s, r)=\mathrm{E}\left(f^{\prime}\left(X_{s}\right) f^{\prime}\left(X_{r}\right) D_{s} D_{r}\right) .
$$

Using dominated convergence, we obtain, when $\beta \rightarrow \infty$,

$$
\int_{0}^{t} \int_{0}^{t} \mu^{\beta}(s, r) \mathrm{d} s \mathrm{~d} r \rightarrow \int_{0}^{t} \int_{0}^{t} \mu(s, r) \mathrm{d} s \mathrm{~d} r .
$$

It is easy to show that

$$
\lim _{\beta \rightarrow \infty} \mathrm{E}\left\{X_{l} \sqrt{\beta}\left(X_{s}^{\beta}-X_{s}\right)\right\}=0, \quad \forall l, s \in[0, t],
$$

so that

$$
\lim _{\beta \rightarrow \infty} \mathrm{E}\left\{\sqrt{\beta}\left(X_{s}^{\beta}-X_{s}\right) \sqrt{\beta}\left(X_{r}^{\beta}-X_{r}\right)\right\}=0, \quad \forall s \neq r \in[0, t] .
$$

Hence $\int_{0}^{t} \int_{0}^{t} \mu(s, r) \mathrm{d} s \mathrm{~d} r=0$ and step 1 follows.

Proof of step 2. Proposition 1 applied to the function $g$ gives, when $\beta \rightarrow \infty$,

$$
\mathrm{E}\left\{J(\beta)^{2}\right\} \rightarrow \sigma^{2} t \mathrm{E}\left\{f^{2}\left(X_{0}\right)\right\},
$$

where $\sigma^{2}=2 \int_{0}^{t} \mathrm{E}\left\{g\left(Y_{0}\right) g\left(Y_{u}\right)\right\} \mathrm{d} u$.

Proof of step 3. Straightforward computations show that the following explicit representation holds:

$$
\begin{aligned}
Y_{s}^{\beta}= & \frac{\sqrt{2 \beta}}{\left(\lambda_{2}^{\beta}-\lambda_{1}^{\beta}\right)}\left\{\lambda_{2}^{\beta} U_{s}^{W}\left(-\lambda_{2}^{\beta}\right)+\frac{\omega}{\sqrt{2}}\left[\exp \left(\lambda_{1}^{\beta} s\right)-\exp \left(\lambda_{2}^{\beta} s\right)\right] X_{0}\right\} \\
& +\frac{\sqrt{2 \beta}}{\left(\lambda_{2}^{\beta}-\lambda_{1}^{\beta}\right)}\left[-\lambda_{1}^{\beta} U_{s}^{W}\left(-\lambda_{1}^{\beta}\right)\right]+\frac{\left[\lambda_{2}^{\beta} \exp \left(\lambda_{2}^{\beta} s\right)-\lambda_{1}^{\beta} \exp \left(\lambda_{1}^{\beta} s\right)\right]}{\left[\lambda_{2}^{\beta}-\lambda_{1}^{\beta}\right]} Y_{0} .
\end{aligned}
$$

Define a new Brownian motion $W^{\beta}$ by

$$
W_{t}^{\beta}=\sqrt{-\lambda_{1}^{\beta}} W\left(\frac{t}{-\lambda_{1}^{\beta}}\right)
$$

and set

$$
Z_{s}^{\beta}=\sqrt{-2 \lambda_{1}^{\beta}} U^{W}\left(\frac{s}{-\lambda_{1}^{\beta}}\right)\left(-\lambda_{1}^{\beta}\right)+\exp (-s) Y_{0}=\sqrt{2} U_{s}^{W^{\beta}}(1)+\exp (-s) Y_{0} .
$$

(For the last identity use $U_{s}^{M}(a)=M_{s}-a \exp (-a s) \int_{0}^{s} \exp (a u) M_{u} \mathrm{~d} u$ ). Now observe that:

$$
\left|\sqrt{\beta} \int_{0}^{t} g\left(Y_{s}^{\beta}\right) \mathrm{d} s-\sqrt{\beta} \int_{0}^{t} g\left(\left[\frac{\lambda_{2}^{\beta}-\lambda_{1}^{\beta}}{\beta}\right] Y_{s}^{\beta}\right) \mathrm{d} s\right| \leqslant\left|\lambda_{2}^{\beta}\right| \sqrt{\frac{2 \pi}{\beta}} \int_{0}^{t}\left|Y_{s}^{\beta}\right| \mathrm{d} s .
$$

But, using Jensen's inequality, when $\beta \rightarrow \infty$, 


$$
\mathrm{E}\left(\frac{1}{\sqrt{\beta}} \int_{0}^{t}\left|Y_{s}^{\beta}\right| \mathrm{d} s\right)^{2} \leqslant \frac{t}{\beta} \int_{0}^{t} \mathrm{E}\left(Y_{s}^{\beta}\right)^{2}=\frac{t^{2}}{\beta} \rightarrow 0 .
$$

Then it will suffice to show that

$$
\sqrt{\beta} \int_{0}^{t}\left(g\left(\frac{\left[\lambda_{2}^{\beta}-\lambda_{1}^{\beta}\right]}{\beta} Y_{s}^{\beta}\right)-g\left(Z_{-\lambda_{1}^{\beta} s}^{\beta}\right)\right) \mathrm{d} s \rightarrow 0 \quad\left(\text { in } L^{2}(\Omega)\right)
$$

to obtain the step.

Let us define

$$
T_{s}^{\beta}=\sqrt{2\left(-\lambda_{2}^{\beta}\right)} U_{\left(\frac{s}{-\lambda_{2}^{\beta}}\right)}^{W}\left(-\lambda_{2}^{\beta}\right)+\exp (-s) X_{0}
$$

Hence we can write

$$
\begin{aligned}
{\left[\frac{\lambda_{2}^{\beta}-\lambda_{1}^{\beta}}{\beta}\right] Y_{s}^{\beta}=} & \frac{1}{\sqrt{\beta}}\left\{-\sqrt{-\lambda_{2}^{\beta}} T_{-\lambda_{2}^{\beta} s}^{\beta}+Z_{-\lambda_{1}^{\beta} s}^{\beta} \sqrt{-\lambda_{1}^{\beta}}\right\}+\frac{\sqrt{-\lambda_{2}^{\beta}}-\omega}{\sqrt{\beta}} \mathrm{e}^{\lambda_{2}^{\beta} s} X_{0} \\
& +X_{0} \frac{\omega \mathrm{e}^{\lambda_{1}^{\beta} s}}{\sqrt{\beta}}+Y_{0} \frac{1}{\sqrt{\beta}}\left(-\sqrt{-\lambda_{1}^{\beta}}-\frac{\lambda_{1}^{\beta}}{\sqrt{\beta}}\right) \mathrm{e}^{\lambda_{1}^{\beta} s}+Y_{0} \frac{\lambda_{2}^{\beta} \mathrm{e}^{\lambda_{2}^{\beta} s}}{\beta} \\
:= & \tilde{Y}_{s}^{\beta}+A_{\beta}(s)+B_{\beta}(s)+C_{\beta}(s)+D_{\beta}(s),
\end{aligned}
$$

where

$$
\begin{gathered}
A_{\beta}(s)=\frac{\sqrt{-\lambda_{2}^{\beta}}-\omega}{\sqrt{\beta}} \mathrm{e}^{\lambda_{2}^{\beta} s} X_{0}, \quad B_{\beta}(s)=X_{0} \frac{\omega \mathrm{e}^{\lambda_{1}^{\beta} s}}{\sqrt{\beta}}, \\
C_{\beta}(s)=Y_{0} \frac{1}{\sqrt{\beta}}\left(-\sqrt{-\lambda_{1}^{\beta}}-\frac{\lambda_{1}^{\beta}}{\sqrt{\beta}}\right) \mathrm{e}^{\lambda_{1}^{\beta} s}, \quad D_{\beta}(s)=Y_{0} \frac{\lambda_{2}^{\beta} \mathrm{e}^{\lambda_{2}^{\beta} s}}{\beta} .
\end{gathered}
$$

Thus,

$$
\begin{aligned}
\sqrt{\beta}\left|\int_{0}^{t}\left(g\left(\frac{\left[\lambda_{2}^{\beta}-\lambda_{1}^{\beta}\right)}{\beta} Y_{s}^{\beta}\right)-g\left(\tilde{Y}_{s}^{\beta}\right)\right) \mathrm{d} s\right| \leqslant \\
\quad \sqrt{\frac{\pi}{2}}\left(\sqrt{\beta} \int_{0}^{t}\left|A_{\beta}(s)\right| \mathrm{d} s+\sqrt{\beta} \int_{0}^{t}\left|B_{\beta}(s)\right| \mathrm{d} s+\sqrt{\beta} \int_{0}^{t}\left|C_{\beta}(s)\right| \mathrm{d} s+\sqrt{\beta} \int_{0}^{t}\left|D_{\beta}(s)\right| \mathrm{d} s\right)
\end{aligned}
$$

therefore it suffices to prove that

(i) $\sqrt{\beta} \int_{0}^{t}\left(g\left(\tilde{Y}_{s}^{\beta}\right)-g\left(Z_{-\lambda_{1}^{\beta} s}^{\beta}\right)\right) \mathrm{d} s \rightarrow 0$,

(ii) $\sqrt{\beta} \int_{0}^{t}\left|A_{\beta}(s)\right| \mathrm{d} s \rightarrow 0, \sqrt{\beta} \int_{0}^{t}\left|B_{\beta}(s)\right| \mathrm{d} s \rightarrow 0$,

$\sqrt{\beta} \int_{0}^{t}\left|C_{\beta}(s)\right| \mathrm{d} s \rightarrow 0, \sqrt{\beta} \int_{0}^{t}\left|D_{\beta}(s)\right| \mathrm{d} s \rightarrow 0$ (in $L^{2}(\Omega)$ ). 
(ii) is an easy consequence of the definition of the terms. To prove (i), observe that

$$
Z_{s}^{\beta}=\sqrt{2} U_{s}^{W^{\beta}}(1)+\exp (-s) Y_{0}
$$

and

$$
T_{s}^{\beta}=\sqrt{2} U_{s}^{\tilde{W}^{\beta}}(1)+\exp (-s) X_{0}
$$

are stationary Ornstein-Uhlenbeck processes of parameter 1 , the former associated with $W_{t}^{\beta}=\sqrt{-\lambda_{1}^{\beta}} W_{\left(t /-\lambda_{1}^{\beta}\right)}$ and the latter associated with $\tilde{W}_{t}^{\beta}=\sqrt{-\lambda_{2}^{\beta}} W_{\left(t /-\lambda_{2}^{\beta}\right)}$.

We also have

$$
\mathrm{E}\left(Z_{-\lambda_{1}^{\beta s}}^{\beta} T_{-\lambda_{2}^{\beta}(s+h)}^{\beta}\right)=\frac{2}{\beta} \mathrm{e}^{\lambda_{2}^{\beta} h}(\exp \{(\beta(h \wedge 0)\}-\exp \{-\beta s\}) .
$$

Finally, it is obvious that:

$$
\lambda_{1}^{\beta}=-\beta+\omega^{2}+O\left(\frac{1}{\beta}\right), \quad \lambda_{2}^{\beta}=-\omega^{2}+O\left(\frac{1}{\beta}\right) .
$$

An elementary computation shows that Lemma 3 (see the Appendix) applies, which proves that (i) holds.

Proof of step 4. After a change of variables, we obtain

$$
C(\beta)_{y, t}=\left(W_{y}, \frac{\sqrt{\beta}}{-\lambda_{1}^{\beta}} \int_{0}^{-\lambda_{1}^{\beta} t} g\left(Z_{s}^{\beta}\right) d s\right) ;
$$

applying the OU inversion formula, we can write $W_{t}^{\beta}=\left(Z_{t}^{\beta}-Y_{0}\right)+\int_{0}^{t} Z_{s}^{\beta} \mathrm{d} s / \sqrt{2}$ and hence,

$$
W_{t}=\frac{1}{\sqrt{-2 \lambda_{1}^{\beta}}}\left(Z_{-\lambda_{1}^{\beta} t}-Y_{0}\right)+\frac{1}{\sqrt{-2 \lambda_{1}^{\beta}}} \int_{0}^{-\lambda_{1}^{\beta} t} Z_{s}^{\beta} \mathrm{d} s .
$$

The first term in the last expression tends to zero in $L^{2}$ as $\beta$ goes to infinity; therefore, $C(\beta)_{y, t}$ has the same asymptotic distribution as

$$
D(\beta)_{y, t}=\frac{1}{\sqrt{-\lambda_{1}^{\beta}}}\left(\frac{1}{\sqrt{2}} \int_{0}^{-\lambda_{1}^{\beta} y} Z_{s}^{\beta} \mathrm{d} s, \int_{0}^{-\lambda_{1}^{\beta} t} g\left(Z_{s}^{\beta}\right) \mathrm{d} s\right) .
$$

But $D(\beta)_{y, t}$ has the same law as

$$
\frac{1}{\sqrt{\beta}}\left(\frac{1}{\sqrt{2}} \int_{0}^{-\lambda_{1}^{\beta} y} Z_{s}^{1} \mathrm{~d} s, \int_{0}^{-\lambda_{1}^{\beta} t} g\left(Z_{s}^{1}\right) \mathrm{d} s\right)
$$

which converges weakly (as a continuous process in $y$, f.d. in $t)$ to $\left(W_{y}, \sigma B_{t}\right)$ because the process $Z^{1}$ is geometrically $\rho$-mixing (see Doukhan 1994, p. 115) and the step is proved. 
Proof of step 5. Write

$$
\left\|V(\beta)-V_{n}(\beta)\right\|_{2}^{2}=\mathrm{E}\left\{V(\beta)^{2}\right\}+\mathrm{E}\left\{V_{n}(\beta)^{2}\right\}-2 \mathrm{E}\left\{V(\beta) V_{n}(\beta)\right\} .
$$

The first term on the right-hand side converges to $\sigma^{2} t E\left\{f\left(X_{0}\right)^{2}\right\}$ as a consequence of proposition 1. For the second term, we have

$$
\mathrm{E}\left\{V_{n}(\beta)^{2}\right\}=\beta \sum_{i=1}^{[n t]} \sum_{j=1}^{[n t]} \mathrm{E}\left\{f\left(X_{\frac{i-1}{n}}^{\beta}\right) f\left(X_{\frac{j-1}{n}}^{\beta}\right) \int_{\frac{i-1}{n}}^{\frac{i}{n}} g\left(Y_{s}^{\beta}\right) \mathrm{d} s \int_{\frac{j-1}{n}}^{\frac{j}{n}} g\left(Y_{s^{\prime}}^{\beta}\right) \mathrm{d} s^{\prime} .\right.
$$

Fix $n$ and $t$ : step 4 implies that

$$
\left(f\left(X_{\frac{i-1}{n}}^{\beta}\right), f\left(X_{\frac{j-1}{n}}^{\beta}\right), \sqrt{\beta} \int_{\frac{i-1}{n}}^{\frac{i}{n}} g\left(Y_{s}^{\beta}\right) \mathrm{d} s, \sqrt{\beta} \int_{\frac{j-1}{n}}^{\frac{j}{n}} g\left(Y_{s^{\prime}}^{\beta}\right) \mathrm{d} s^{\prime}\right)
$$

converges in law, as $\beta$ goes to infinity, to

$$
\left(f\left(X_{\frac{i-1}{n}}\right), f\left(X_{\frac{j-1}{n}}\right), \sigma\left(B_{\frac{i}{n}}-B_{\frac{i-1}{n}}\right), \sigma\left(B_{\frac{j}{n}}-B_{\frac{j-1}{n}}\right)\right) .
$$

On the other hand, by Hölder,

$$
f\left(X_{\frac{i-1}{n}}^{\beta}\right) f\left(X_{\frac{j-1}{n}}^{\beta}\right) \int_{\frac{i-1}{n}}^{\frac{i}{n}} g\left(Y_{s}^{\beta}\right) \mathrm{d} s \int_{\frac{j-1}{n}}^{\frac{j}{n}} g\left(Y_{s^{\prime}}^{\beta}\right) \mathrm{d} s
$$

is bounded in $L^{2}(\Omega)$, hence it is uniformly integrable and we conclude that, for $\beta$ going to infinity, $E\left\{V_{n}(\beta)^{2}\right\}$ converges to

$$
\sigma^{2} \sum_{i=1}^{[n t]} \mathrm{E}\left\{f^{2}\left(X_{\frac{i-1}{n}}\right)\right\} \frac{1}{n} \approx \sigma^{2} t \mathrm{E}\left\{f\left(X_{0}\right)^{2}\right\} .
$$

Finally, Lemma 4 (see the Appendix) shows that $\mathrm{E}\left\{V(\beta) V_{n}(\beta)\right\}$ converges to $\sigma^{2} t \mathrm{E}\left\{f\left(X_{0}\right)^{2}\right\}$ if $\beta$ and $n$ converge to infinity (in that order), which concludes the proof.

\section{Appendix}

Lemma 2. With the notation of Proposition 1, define:

$$
K_{2}(\beta):=2 \beta \int_{M / \beta}^{t}(t-s) \mathrm{E}\left\{f\left(X_{0}\right) f\left(X_{s}^{\beta}\right) h\left(Y_{0}\right) h\left(Y_{s}^{\beta}\right)\right\} .
$$

Then

$$
\lim _{M \rightarrow \infty} \limsup _{\beta \rightarrow \infty}\left|K_{2}(\beta)\right|=0
$$

Proof. Let us start by introducing some notation. Write 


$$
\zeta_{\beta}=\sqrt{1-\frac{\omega^{2}}{\beta}} \approx 1-\frac{\omega^{2}}{2 \beta} .
$$

Then, using the fact that $X_{0}^{\beta}=X_{0}, Y_{0}^{\beta}=Y_{0}$, we obtain, for all $s \geqslant 0$,

$$
\begin{aligned}
& \mathrm{E}\left\{X_{0} X_{s}^{\beta}\right\}=\rho^{\beta}(s)=\frac{1}{2 \zeta_{\beta}}\left\{\left(1+\zeta_{\beta}\right) \mathrm{e}^{-\frac{\beta}{2}\left(1-\xi_{\beta}\right) s}-\left(1-\zeta_{\beta}\right) \mathrm{e}^{-\frac{\beta}{2}\left(1+\zeta_{\beta}\right) s}\right\} \\
& \mathrm{E}\left\{X_{0} Y_{s}^{\beta}\right\}=\rho_{1}^{\beta}(s)=\frac{\omega}{\sqrt{\beta} \xi_{\beta}}\left\{\mathrm{e}^{-\frac{\beta}{2}\left(1+\zeta_{\beta}\right) s}-\mathrm{e}^{-\frac{\beta}{2}\left(1-\zeta_{\beta}\right) s}\right\} \\
& \mathrm{E}\left\{Y_{0} Y_{s}^{\beta}\right\}=\rho_{2}^{\beta}(s)=\frac{1}{2 \zeta_{\beta}}\left\{\left(1+\zeta_{\beta}\right) \mathrm{e}^{-\frac{\beta}{2}\left(1+\zeta_{\beta}\right) s}-\left(1-\zeta_{\beta}\right) \mathrm{e}^{-\frac{\beta}{2}\left(1-\zeta_{\beta}\right) s}\right\} .
\end{aligned}
$$

We can decompose

$$
\begin{aligned}
& X_{0}=Z_{0}(\beta)+A_{1}(\beta) Y_{0}+A_{2}(\beta) Y_{s}^{\beta} \\
& X_{s}^{\beta}=Z_{1}(\beta)+B_{1}(\beta) Y_{0}+B_{2}(\beta) Y_{s}^{\beta},
\end{aligned}
$$

with $\left(Z_{0}(\beta), Z_{1}(\beta)\right)$ independent of $\left(Y_{0}, Y_{s}^{\beta}\right)$. The coefficients are given by

$$
\begin{array}{cc}
A_{1}(\beta)=\frac{-\rho_{2}^{\beta}(s) \rho_{1}^{\beta}(s)}{1-\left(\rho_{2}^{\beta}(s)\right)^{2}}, & A_{2}(\beta)=\frac{\rho_{1}^{\beta}(s)}{1-\left(\rho_{2}^{\beta}(s)\right)^{2}} \\
B_{1}(\beta)=-A_{2}(\beta), & B_{2}(\beta)=-A_{1}(\beta) ;
\end{array}
$$

it is easy to verify that if $M>\log 2, s>2 M / \beta$, we have

$$
A_{i}(\beta)=O(1 / \sqrt{\beta}), B_{i}(\beta)=O(1 / \sqrt{\beta}), i=1,2 .
$$

We also obtain

$$
\begin{gathered}
\lim _{\beta \rightarrow \infty} \sqrt{\beta} A_{1}(\beta)=0, \quad \lim _{\beta \rightarrow \infty} \sqrt{\beta} A_{2}(\beta)=-\omega \mathrm{e}^{-\omega^{2} s}, \\
\lim _{\beta \rightarrow \infty} \sqrt{\beta} B_{1}(\beta)=\omega \mathrm{e}^{-\omega^{2} s}, \quad \lim _{\beta \rightarrow \infty} B_{2}(\beta)=0 .
\end{gathered}
$$

Thus, we have that

$$
\begin{gathered}
\mathrm{E}\left\{\left(Z_{1}(\beta)-X_{s}^{\beta}\right)^{2}\right\}=O\left(\frac{1}{\beta}\right), \quad \mathrm{E}\left\{\left(Z_{0}(\beta)-X_{0}\right)^{2}\right\}=O\left(\frac{1}{\beta}\right), \\
\mathrm{E}\left\{\left(Z_{1}(\beta)\right)^{2}\right\}=O(1), \quad \mathrm{E}\left\{\left(Z_{0}(\beta)\right)^{2}\right\}=O(1) .
\end{gathered}
$$

Observe that if $\theta_{0}(\beta)$ is between $X_{0}$ and $Z_{0}(\beta)$, and $\theta_{1}(\beta)$ is between $X_{s}^{\beta}$ and $Z_{1}(\beta)$, then

$$
\mathrm{E}\left\{\left(\theta_{0}(\beta)-X_{0}\right)^{2}\right\}=O\left(\frac{1}{\beta}\right), \quad \mathrm{E}\left\{\left(\theta_{1}(\beta)-X_{s}^{\beta}\right)^{2}\right\}=O\left(\frac{1}{\beta}\right) .
$$

Using the Taylor series

$$
f\left(X_{0}\right)=f\left(Z_{0}(\beta)\right)+f^{\prime}\left(Z_{0}(\beta)\right)\left(A_{1}(\beta) Y_{0}+A_{2}(\beta) Y_{s}^{\beta}\right)+\frac{1}{2} f^{\prime \prime}\left(\theta_{0}(\beta)\right)\left(A_{1}(\beta) Y_{0}+A_{2}(\beta) Y_{s}^{\beta}\right)^{2}
$$




$$
f\left(X_{s}^{\beta}\right)=f\left(Z_{1}(\beta)\right)+f^{\prime}\left(Z_{1}(\beta)\right)\left(B_{1}(\beta) Y_{0}+B_{2}(\beta) Y_{s}^{\beta}\right)+\frac{1}{2} f^{\prime \prime}\left(\theta_{1}(\beta)\right)\left(B_{1}(\beta) Y_{0}+B_{2}(\beta) Y_{s}^{\beta}\right)^{2},
$$

we can decompose

$$
2 \beta \int_{\frac{2 M}{\beta}}^{t}(t-s) \mathrm{E}\left\{f\left(X_{0}\right) f\left(X_{s}^{\beta}\right) h\left(Y_{0}\right) h\left(Y_{s}^{\beta}\right)\right\} \mathrm{d} s
$$

as the sum of nine terms of six different types:

Type 1. We have two terms of the type $2 \beta \int_{\frac{2 M}{\beta}}^{t}(t-s) \mathrm{E}\left\{f\left(Z_{1}(\beta)\right) f^{\prime}\left(Z_{0}(\beta)\right)\right\}\left\{A_{1}(\beta) \mathrm{E}\left(Y_{0} h\left(Y_{0}\right) h\left(Y_{s}^{\beta}\right)\right)+A_{2}(\beta) \mathrm{E}\left(Y_{s}^{\beta} h\left(Y_{0}\right) h\left(Y_{s}^{\beta}\right)\right)\right\} \mathrm{d} s=0$, because (by symmetry), if $(u, v)$ is a centred Gaussian vector, then $\mathrm{E}(U h(U) h(V))=0$. Type 2. There is one term of the type

$$
\begin{aligned}
2 \beta \int_{\frac{2 M}{\beta}}^{t}( & -s) \mathrm{E}\left\{f^{\prime}\left(Z_{0}(\beta)\right) f^{\prime}\left(Z_{1}(\beta)\right)\right\} \mathrm{E}\left\{\left(A_{1}(\beta) Y_{0}\right.\right. \\
& \left.\left.+A_{2}(\beta) Y_{s}^{\beta}\right)\left(B_{1}(\beta) Y_{0}+B_{2}(\beta) Y_{s}^{\beta}\right) h\left(Y_{0}\right) h\left(Y_{s}^{\beta}\right)\right\} \mathrm{d} s
\end{aligned}
$$

the integrand is bounded by (A.1), and it converges with $\beta$ to

$$
(t-s) \mathrm{E}\left\{f^{\prime}\left(X_{0}\right) f^{\prime}\left(X_{s}\right)\left(\omega \mathrm{e}^{-\omega^{2} s}\right) h\left(Y_{0}\right)\right\} \mathrm{E}\left\{-\omega \mathrm{e}^{-\omega^{2} s} V h(V)\right\}=0
$$

(because $\left(X_{0}, X_{s}, Y_{0}, V\right)$ is Gaussian and $V$ is independent of $\left.\left(X_{0}, X_{s}, Y_{0}\right)\right)$; hence, by dominated convergence, this term goes to zero.

Type 3. There are two terms of the type

$$
\begin{gathered}
2 \beta \int_{\frac{2 M}{\beta}}^{t}(t-s) \mathrm{E}\left\{f ^ { \prime } ( Z _ { 1 } ( \beta ) ) ( B _ { 1 } ( \beta ) Y _ { 0 } + B _ { 2 } ( \beta ) Y _ { s } ^ { \beta } ) \frac { 1 } { 2 } f ^ { \prime \prime } ( \theta _ { 0 } ( \beta ) ) \left(A_{1}(\beta) Y_{0}\right.\right. \\
\left.\left.\quad+A_{2}(\beta) Y_{s}^{\beta}\right)^{2} h\left(Y_{0}\right) h\left(Y_{s}^{\beta}\right)\right\} \mathrm{d} s=O\left(\frac{1}{\sqrt{\beta}}\right)
\end{gathered}
$$

these two terms go to zero.

Type 4. We obtain two terms of the type

$$
2 \beta \int_{\frac{2 M}{\beta}}^{t}(t-s) \mathrm{E}\left\{f\left(Z_{1}(\beta)\right) \frac{1}{2} f^{\prime \prime}\left(\theta_{0}(\beta)\right)\left(A_{1}(\beta) Y_{0}+A_{2}(\beta) Y_{s}^{\beta}\right)^{2} h\left(Y_{0}\right) h\left(Y_{s}^{\beta}\right)\right\} \mathrm{d} s
$$

by (A.1) and dominated convergence it is easy to see that this integral converges with $\beta$ to

$$
2 \int_{0}^{t}(t-s) \mathrm{E}\left\{f\left(X_{s}\right) \frac{1}{2} f^{\prime \prime}\left(X_{0}\right) h\left(Y_{0}\right)\right\} \mathrm{E}\left\{-\omega^{2} \mathrm{e}^{-\omega^{2} s} V h(V)\right\} \mathrm{d} s=0,
$$

therefore these two terms go to zero. 
Type 5. We also have one term of the type

$$
\begin{gathered}
\int_{\frac{2 M}{\beta}}^{t}(t-s) \mathrm{E}\left\{\frac { 1 } { 2 } f ^ { \prime \prime } ( \theta _ { 1 } ( \beta ) ) ( B _ { 1 } ( \beta ) Y _ { 0 } + B _ { 2 } ( \beta ) Y _ { s } ^ { \beta } ) ^ { 2 } \frac { 1 } { 2 } f ^ { \prime \prime } ( \theta _ { 0 } ( \beta ) ) \left(A_{1}(\beta) Y_{0}\right.\right. \\
\left.\left.+A_{2}(\beta) Y_{s}^{\beta}\right)^{2} h\left(Y_{0}\right) h\left(Y_{s}^{\beta}\right)\right\} \mathrm{d} s=O\left(\frac{1}{\sqrt{\beta}}\right) ;
\end{gathered}
$$

this term is also negligible.

Type 6. The remaining term is

$$
\int_{\frac{2 M}{\beta}}^{t} \frac{2}{\beta}(t-s) \mathrm{E}\left\{f\left(Z_{0}(\beta)\right) f\left(Z_{1}(\beta)\right) h\left(Y_{0}\right) h\left(Y_{s}^{\beta}\right)\right\} \mathrm{d} s:=I_{\beta} .
$$

We conclude that

$$
\limsup _{\beta \rightarrow \infty}\left|K_{2}(\beta)\right|=\limsup _{\beta \rightarrow \infty}\left|2 \beta I_{\beta}\right| ;
$$

using the independence and Hölder we deduce that

$$
I_{\beta} \leqslant C t \beta \int_{\frac{2 M}{\beta}}^{t}\left|\mathrm{E}\left\{h\left(Y_{0}\right) h\left(Y_{s}^{\beta}\right)\right\}\right| \mathrm{d} s .
$$

Therefore, it suffices to show that

$$
\lim _{M \rightarrow \infty} \limsup _{\beta \rightarrow \infty} \int_{\frac{2 M}{\beta}}^{t}\left|\mathrm{E}\left\{h\left(Y_{0}\right) h\left(Y_{s}^{\beta}\right)\right\}\right| \mathrm{d} s=0 .
$$

By Mehler's formula

$$
\left|\mathrm{E}\left\{h\left(Y_{0}\right) h\left(Y_{s}^{\beta}\right)\right\}\right| \leqslant \sum_{n=1}^{\infty} \hat{h}_{2 n}^{2}(2 n) !\left(\rho_{2}^{\beta}(s)\right)^{2 n} \leqslant\left(\sum_{n=1}^{\infty} \hat{h}_{2 n}^{2}(2 n) !\right)\left(\rho_{2}^{\beta}(s)\right)^{2} .
$$

Hence, it suffices to prove that

$$
\lim _{M \rightarrow \infty} \limsup _{\beta \rightarrow \infty} 2 \beta \int_{\frac{2 M}{\beta}}^{t}\left(\rho_{2}^{\beta}(s)\right)^{2} \mathrm{~d} s=0 ;
$$

but this is an easy consequence of the definition of $\rho_{2}^{\beta}(s)$.

Lemma 3. Let $a, b, c$, $d$ denote real functions. Let $\xi^{\beta}=\left(\xi_{s}^{\beta} \geqslant 0\right)$ and $\xi^{\beta}=\left(\xi_{s}^{\beta} \geqslant 0\right)$ be two stationary Ornstein-Uhlenbeck processes of parameter 1, such that:

$$
\mathrm{E}\left(\xi_{s+h}^{\beta} \zeta_{\beta s}^{\beta}\right)=c(\beta) \exp (-h)(\exp \{d(\beta) \beta(h \wedge 0)\}-\exp \{d(\beta) \beta s\})
$$

Define

$$
\tau_{s}^{\beta}=a(\beta) \xi_{s}^{\beta}+b(\beta) \xi_{\beta s}^{\beta} .
$$


Assume that, for $\beta$ going to infinity,

$$
\begin{gathered}
a(\beta)=O\left(\frac{1}{\sqrt{\beta}}\right), \quad b(\beta)=1+O\left(\frac{1}{\sqrt{\beta}}\right), \\
c(\beta)=c+O\left(\frac{1}{\beta}\right), \quad d(\beta)=1-\frac{\alpha}{\beta}+O\left(\frac{1}{\beta^{2}}\right),
\end{gathered}
$$

where $a, c>0$.

Then

$$
\sqrt{\beta} \int_{0}^{t}\left(g\left(\tau_{s}^{\beta}\right)-g\left(\xi_{\beta s}^{\beta}\right)\right) \mathrm{d} s \rightarrow 0 \quad \text { in } L^{2}(\Omega) .
$$

Proof. We have

$$
\begin{aligned}
\mathrm{E}\left[\left\{\sqrt{\beta} \int_{0}^{t}\left(g\left(\tau_{s}^{\beta}\right)-g\left(\xi_{\beta s}^{\beta}\right)\right) \mathrm{d} s\right\}^{2}\right]= & \frac{\pi}{2} \int_{0}^{t} \int_{0}^{t} \beta\left[\left(\mathrm{E}\left\{\left|\tau_{s}^{\beta} \| \tau_{x}^{\beta}\right|\right\}-\mathrm{E}\left\{\left|\xi_{\beta s}^{\beta} \| \tau_{x}^{\beta}\right|\right\}\right.\right. \\
& \left.-\mathrm{E}\left\{\left|\xi_{\beta x}^{\beta} \| \tau_{s}^{\beta}\right|\right\}+\mathrm{E}\left(\left|\xi_{\beta s}^{\beta} \| \xi_{\beta x}^{\beta}\right|\right)\right] \mathrm{d} s \mathrm{~d} x
\end{aligned}
$$

To compute this integral we use the elementary fact that if

$$
(X, Y) \sim N\left(0,\left(\begin{array}{ll}
1 & \rho \\
\rho & 1
\end{array}\right)\right)
$$

then

$$
\mathrm{E}(|X \| Y|)=2 \frac{\sqrt{1-\rho^{2}}}{\pi}+\frac{2|\rho|}{\pi} \arcsin |\rho|=F(\rho) .
$$

Taking into account that $\xi^{\beta}, \xi^{\beta}$ are stationary Ornstein-Uhlenbeck processes of parameter 1, and using (A.2), we obtain:

$\operatorname{cov}\left(\tau_{s}^{\beta}, \tau_{x}^{\beta}\right)=a(\beta)^{2} \exp (-|x-s|)+b(\beta)^{2} \exp (-\beta|x-s|)+a(\beta) b(\beta)\left(R_{\beta}(s, x-s)+R_{\beta}(x, s-x)\right)$, where

$$
R_{\beta}(s, h):=\mathrm{E}\left(\xi_{\beta s}^{\beta} \xi_{s+h}^{\beta}\right)=c(\beta) \exp (-h)(\exp \{d(\beta) \beta(h \wedge 0)\}-\exp \{d(\beta) \beta s\}) .
$$

In particular,

$$
v_{\beta}^{2}(s):=\operatorname{var}\left(\tau_{s}^{\beta}\right)=a(\beta)^{2}+b(\beta)^{2}+2 a(\beta) b(\beta)(1-\exp (-d(\beta) \beta s)) .
$$

Therefore, we have

$$
\begin{array}{cc}
\mathrm{E}\left\{\left|\xi_{\beta s}^{\beta} \| \xi_{\beta x}^{\beta}\right|\right\}=F\left(\Gamma_{\beta}^{1}(s, x)\right), & \mathrm{E}\left\{\left|\xi_{\beta s}^{\beta} \| \tau_{x}^{\beta}\right|\right\}=v_{\beta}(x) F\left(\Gamma_{\beta}^{3}(s, x)\right), \\
\mathrm{E}\left\{\left|\xi_{\beta x}^{\beta} \| \tau_{s}^{\beta}\right|\right\}=v_{\beta}(s) F\left(\Gamma_{\beta}^{4}(s, x)\right), & \mathrm{E}\left\{\left|\tau_{s}^{\beta} \| \tau_{x}^{\beta}\right|\right\}=v_{\beta}(s) v_{\beta}(x) F\left(\Gamma_{\beta}^{2}(s, x)\right),
\end{array}
$$

where 
$\Gamma_{\beta}^{1}(s, x):=\exp (-\beta|x-s|)$

$\Gamma_{\beta}^{2}(s, x):=\frac{a(\beta)^{2} \exp (-|x-s|)+b(\beta)^{2} \exp (-\beta|x-s|)+a(\beta) b(\beta)\left(R_{\beta}(s, x-s)+R_{\beta}(x, s-x)\right)}{v_{\beta}(s) v_{\beta}(x)}$,

$\Gamma_{\beta}^{3}(s, x):=\frac{a(\beta) R_{\beta}(s, x-s)+b(\beta) \exp (-\beta|x-s|)}{v_{\beta}(x)}$,

$\Gamma_{\beta}^{4}(s, x):=\Gamma_{\beta}^{3}(x, s)$.

We split the integral according to whether $s \leqslant x$ or not. We will only consider the case $s \leqslant x$ because the other is completely similar. Calling the integrand $\varphi_{\beta}(s, x)$, we obtain

$$
\begin{aligned}
I(\beta) & :=\int_{s \leqslant x} \varphi_{\beta}(s, x) \mathrm{d} s \mathrm{~d} x=\int_{0}^{t} \int_{s}^{s+\varepsilon / \beta} \varphi_{\beta}(s, x) \mathrm{d} s \mathrm{~d} x+\int_{0}^{t} \int_{s+\varepsilon / \beta}^{t} \varphi_{\beta}(s, x) \mathrm{d} s \mathrm{~d} x \\
& :=I_{\beta}(1)+I_{\beta}(2) .
\end{aligned}
$$

Since the function $F$ is bounded by unity, we obtain

$$
\left|I_{\beta}(1)\right| \leqslant C t \varepsilon .
$$

Fix $\varepsilon>0$; it suffices to show that $I_{\beta}(2) \rightarrow 0$ when $\beta \rightarrow+\infty$.

An elementary calculation shows that for $x \geqslant s+\varepsilon / \beta$, we have

$$
\max _{1 \leqslant n \leqslant 4} \sup _{\left\{(s, x): t \geqslant s \geqslant \frac{\varepsilon}{\beta}, x \geqslant 0\right\}} \Gamma_{\beta}^{n}(s, x) \leqslant \Gamma(\varepsilon)<1 \quad \forall \beta \geqslant \beta_{0} .
$$

Therefore we use the second-order Taylor's expansion of $F$,

$$
F(\rho)=\frac{\pi}{2}+M(\rho) \rho^{2},
$$

where $M(\rho)$ is uniformly bounded by $M$ for $|\rho| \leqslant \Gamma(\varepsilon)$.

Thus, we have

$$
\varphi_{\beta}(s, x) \leqslant M\left\{\left(\Gamma_{\beta}^{1}(s, x)\right)^{2}+v_{\beta}(x)\left(\Gamma_{\beta}^{3}(s, x)\right)^{2}+v_{\beta}(s)\left(\Gamma_{\beta}^{3}(x, s)\right)^{2}+v_{\beta}(s) v_{\beta}(x)\left(\Gamma_{\beta}^{2}(s, x)\right)^{2}\right\} .
$$

It follows that:

(a) $\varphi_{\beta}$ is uiformly bounded for $s \geqslant x+\varepsilon / \beta$ and $\beta$ big enough

(b) $\varphi_{\beta}(s, x)$ converges to zero when $\beta$ goes to infinity, for $s<x$.

An application of dominated convergence concludes the proof.

Lemma 4. With the notation of Theorem 2, define

$$
V(\beta)=\sqrt{\beta} \int_{0}^{t} f\left(X_{s}^{\beta}\right) g\left(Y_{s}^{\beta}\right) \mathrm{d} s
$$

and its discretization 


$$
V_{n}(\beta)=\sqrt{\beta} \sum_{n=1}^{[n t]} f\left(X_{\frac{i-1}{n}}^{\beta}\right) \int_{\frac{i-1}{n}}^{\frac{i}{n}} g\left(Y_{s}^{\beta}\right) \mathrm{d} s .
$$

Then

$$
\lim _{n \rightarrow \infty} \lim _{\beta \rightarrow \infty} \mathrm{E}\left\{V(\beta) V_{n}(\beta)\right\}=\sigma^{2} t \mathrm{E}\left\{f\left(X_{0}\right)^{2}\right\}
$$

Proof. Given $m$ a positive integer and $h \in L^{2}(\mu)$ a continuous real function, define

$$
h_{m}(x)=\sum_{l=1}^{l=m} \hat{h}(l) H_{l}(x) \text {. }
$$

Consider

$$
\begin{gathered}
V^{M, m}(\beta)=\sqrt{\beta} \int_{0}^{t} f_{m}\left(X_{s}^{\beta}\right) g_{M}\left(Y_{s}^{\beta}\right) \mathrm{d} s, \\
V_{n}^{M, m}(\beta)=\sqrt{\beta} \sum_{n=1}^{[n t]} f_{m}\left(X_{\frac{i-1}{n}}^{\beta}\right) \int_{\frac{i-1}{n}}^{\frac{i}{n}} g_{M}\left(Y_{s}^{\beta}\right) \mathrm{d} s .
\end{gathered}
$$

Since

$$
\begin{aligned}
\mathrm{E}\left\{\left|V(\beta) V_{n}(\beta)-V^{M, m}(\beta) V_{n}^{M, m}(\beta)\right|\right\} \leqslant & \left(\mathrm{E}\left[\left(V_{n}(\beta)-V_{n}^{M, m}(\beta)\right)^{2}\right] \mathrm{E}\left[V(\beta)^{2}\right]\right)^{\frac{1}{2}} \\
& +\left(\mathrm{E}\left[\left(V(\beta)-V^{M, m}(\beta)\right)^{2}\right] \mathrm{E}\left[V_{n}^{M, m}(\beta)^{2}\right]\right)^{\frac{1}{2}},
\end{aligned}
$$

it suffices to show that

(i) $\lim _{M, m \rightarrow \infty} \lim _{n \rightarrow \infty} \lim _{\beta \rightarrow \infty} \mathrm{E}\left[\left(V_{n}(\beta)-V_{n}^{M, m}(\beta)\right)^{2}\right]=0$;

(ii) $\lim _{M, m \rightarrow \infty} \lim _{\beta \rightarrow \infty} \mathrm{E}\left[\left(V(\beta)-V^{M, m}(\beta)\right)^{2}\right]=0$;

(iii) $\limsup _{M, m \rightarrow \infty} \limsup \limsup \mathrm{E}\left[\left(V_{n}^{M, m}(\beta)^{2}\right]<\infty\right.$,

(iv) $\lim _{M, m \rightarrow \infty} \lim _{n \rightarrow \infty} \lim _{\beta \rightarrow \infty} \mathrm{E}\left\{V^{M, m}(\beta) V_{n}^{M, m}(\beta)\right\}=\sigma^{2} t \mathrm{E}\left\{f\left(X_{0}\right)^{2}\right\}$.

This follows from an application of the diagram formula (see Breuer and Major 1983) and the orthogonality conditions for the random variables $\left(X_{0}(\beta), Y_{0}(\beta), X_{s}^{\beta}, Y_{s}^{\beta}\right)$; for the details, we refer to Lemmas 14 and 15 of Berzin et al. (1998).

\section{References}

Azaïs, J.-M. (1989) Approximation des trajectoires et temps local des diffusions. Ann. Inst. Henri Poincaré Probab. Statist., 25, 175-194.

Azaïs, J.-M. and Florens-Zmirou, D. (1987) Approximation du temps local des processus gaussiens stationnaires par régularisation des trajectories. Probab. Theory Related Fields, 76, 121-132.

Berman, S.M. (1969) Local times and sample properties of stationary Gaussian processes. Trans. Amer. Math. Soc., 137, 277-299. 
Berzin, C., León, J.R. and Ortega, J. (1998) Level crossings and local time for regularized Gaussian process. Probab. Math. Statist., 18, 19-61.

Borodin, A.N. (1989) Brownian local time. Russian Math. Surveys, 44(2), 1-51.

Breuer, P. and Major, P. (1983) Central theorems for non-linear functionals of Gaussian fields. $J$. Multivariate Anal., 13, 425-441.

Doukhan, P. (1994) Mixing: Properties and Examples, Lectures Notes in Statist. 85. Berlin: SpringerVerlag.

Jacobsen, M. (1996) Laplace and the origin of the Ornstein-Uhlenbeck process. Bernoulli, 2, 271286.

Karatzas, I. and Shreve, S.E. (1991) Brownian Motion and Stochastic Calculus, 2nd edition. Berlin: Springer-Verlag.

Nelson, E. (1968) Dynamical Theories of Brownian Motion. Mathematical Notes. Princeton, NJ: Princeton University Press.

Nualart, D. and Wschebor, M. (1991) Intégration par parties dans l'espace de Wiener et approximation du temps local. Probab. Theory Related Fields, 90, 83-109.

Revuz, D. and Yor, M. (1991) Continuous Martingales and Brownian Motion. Berlin: Springer-Verlag. Rice, O. (1945) Mathematical analysis of random noise. Bell Syst. Tech. J., 24, 46-156.

Wang, S. and Uhlenbeck, G.E. (1945) On the theory of the Brownian motion II. Rev. Modern Phys., 17, 323-342.

Wschebor, M. (1985) Surfaces Aléatoires: Mesure Géométrique des Ensembles de Niveau, Lecture Notes in Math. 1147. Berlin: Springer-Verlag.

Received April 1997 and revised October 1998 